\title{
Computed tomography angiography in patients with active gastrointestinal bleeding*
}

\author{
Angiotomografia abdominal no paciente com sangramento gastrintestinal ativo
}

\section{Fatima Regina Silva Reis ${ }^{1}$, Patricia Prando Cardia ${ }^{2}$, Giuseppe D'Ippolito ${ }^{3}$}

Reis FRS, Cardia PP, D'Ippolito G. Computed tomography angiography in patients with active gastrointestinal bleeding. Radiol Bras. 2015 Nov/Dez;48(6): 381-390.

Abstract Gastrointestinal bleeding represents a common medical emergency, with considerable morbidity and mortality rates, and a prompt diagnosis is essential for a better prognosis. In such a context, endoscopy is the main diagnostic tool; however, in cases where the gastrointestinal hemorrhage is massive, the exact bleeding site might go undetected. In addition, a trained professional is not always present to perform the procedure. In an emergency setting, optical colonoscopy presents limitations connected with the absence of bowel preparation, so most of the small bowel cannot be assessed. Scintigraphy cannot accurately demonstrate the anatomic location of the bleeding and is not available at emergency settings. The use of capsule endoscopy is inappropriate in the acute setting, particularly in the emergency department at night, and is a highly expensive method. Digital angiography, despite its high sensitivity, is invasive, presents catheterizationrelated risks, in addition to its low availability at emergency settings. On the other hand, computed tomography angiography is fast, widely available and minimally invasive, emerging as a promising method in the diagnostic algorithm of these patients, being capable of determining the location and cause of bleeding with high accuracy. Based on a critical literature review and on their own experience, the authors propose a computed tomography angiography protocol to assess the patient with gastrointestinal bleeding.

Keywords: Gastrointestinal hemorrhage; Computed tomography; Angiography; Multidetector computed tomography.

Res u mo O sangramento gastrintestinal representa uma emergência médica comum, com taxas consideráveis de morbimortalidade, sendo essencial um rápido diagnóstico para se obter um prognóstico favorável. A endoscopia digestiva alta é o principal método diagnóstico, porém, em casos de sangramento maciço, o exato local de sangramento pode não ser detectável. Além disso, nem sempre está disponível um profissional treinado para a sua execução. A colonoscopia óptica na urgência tem suas limitações ligadas à ausência do preparo intestinal adequado e não permite avaliar a maior parte do intestino delgado. A cintilografia não determina a localização precisa do sangramento e não é disponível na emergência. O uso da cápsula endoscópica é inapropriado no cenário emergencial e tem custo elevado. A angiografia digital, apesar de altamente sensível, é um método invasivo, tem riscos associados ao cateterismo, além de baixa disponibilidade emergencial. Por outro lado, pela sua rapidez, ampla disponibilidade e baixa invasividade, a angiotomografia desponta como opção promissora no algoritmo diagnóstico desses pacientes, sendo capaz de determinar o local e a causa do sangramento com alta acurácia, bem como orientar o seu tratamento. Com base em revisão crítica da literatura e na nossa própria experiência, propomos um protocolo de exame de angiotomografia para o paciente com hemorragia digestiva.

Unitermos: Hemorragia gastrintestinal; Tomografia computadorizada; Angiografia; Tomografia computadorizada multidetectores.

\section{INTRODUCTION}

Gastrointestinal bleeding (GIB) represents a common medical emergency, with a yearly incidence of $40-150$ cases/ 100,000 people presenting with upper GIB and 20-27 cases/

* Study developed in the Department of Imaging Diagnosis at Escola Paulista de Medicina - Universidade Federal de São Paulo (EPM-Unifesp), São Paulo, SP, Brazil.

1. Master, Professional Mastership Program in association with Medical Residency (Meparem), MD, Radiologist, Department of Imaging Diagnosis, Escola Paulista de Medicina - Universidade Federal de São Paulo (EPM-Unifesp), São Paulo, SP, Brazil.

2. PhD, MD, Radiologist, Centro Radiológico Campinas, Hospital Vera Cruz, Campinas, SP, Brazil.

3. Private Docent, Associate Professor, Department of Imaging Diagnosis, Escola Paulista de Medicina - Universidade Federal de São Paulo (EPM-Unifesp), São Paulo, SP, Brazil.

Mailing Address: Dra. Fatima Regina Silva Reis. Rua Jorge Chammas, 224, ap. 41, Torre Centenary, Vila Mariana. São Paulo, SP, Brazil, 04016-070. E-mail: fatima.rsreis@gmail.com.

Received February 25, 2014. Accepted after revision July 18, 2014.
100,000 people presenting with lower $\mathrm{GIB}^{(1)}$, and is a common cause of admission at hospital emergency services. Among its main causes, one should highlight esophageal and gastric ulcers $^{(2)}$. GIB is classified either as upper or lower, depending upon its origin above or below the ligament of Treitz, respectively. Approximately $75 \%$ of the patients presenting with acute GIB are upper GIB cases ${ }^{(3)}$. Obscure GIB is defined as bleeding with undetermined causes following upper gastrointestinal endoscopy, optical colonoscopy and small bowel radiological evaluation. It can be occult, when detected only by laboratory fecal occult blood tests, or overt, when it clinically manifests as active bleeding due to hematemesis, melena, hematochezia or enterorrhagia, frequently leading patients to seek emergency care, and possibly causing hemodynamic instability ${ }^{(4)}$.

In $75 \%$ of the cases, the bleeding ceases spontaneously, but recurrence is observed $25 \%$ of cases, causing mortality 
in $8 \%$ to $14 \%$ of cases, sometimes reaching $40 \%$ in hemodynamically unstable patients ${ }^{(5)}$. In addition, intermittence in GIB is frequently observed, impairing the identification of its cause. For that reason, a prompt diagnosis is essential for a favorable prognosis for such patients ${ }^{(3)}$. In such a context, the role played by the radiologist is that of identifying, characterizing and, whenever pertinent, treating the bleeding lesion.

\section{DIAGNOSTIC APPROACH STRATEGIES}

The methods involved in the diagnosis of GIB include upper gastrointestinal endoscopy, optical colonoscopy, capsule endoscopy, scintigraphy, digital angiography and computed tomography (CT), most recently utilizing multiple detectors (MDCT) ${ }^{(6)}$

Upper gastrointestinal endoscopy is the main diagnostic tool in upper GIB. It is a safe and widely available procedure, during which biopsies and treatment of vessels with active bleedings can be concomitantly performed, also with treatment of vessels presenting with a risk for bleeding. It has a reported sensitivity of 92-98\% and specificity of 33$100 \%^{(7-9)}$. However, it is an invasive method, presenting with risk of perforations and with limited effectiveness in those cases of massive hemorrhage where large amounts of blood and presence of blood clots may impair the detection of the bleeding site ${ }^{(10,11)}$. In at least one series, upper gastrointestinal endoscopy was not diagnostic in $24 \%$ of cases of upper gastrointestinal hemorrhage ${ }^{(12)}$.

Optical colonoscopy is many times the first diagnostic method utilized in patients with lower GIB, with a sensitivity of approximately $50 \%$ for the detection of both the location and cause of the bleeding ${ }^{(13)}$. It is a widely accessible method, and allows for local treatment and biopsies. However, its implementation at an emergency scenario poses some obstacles such as the need for bowel preparation and the inappropriate visualization of the colon due to the presence of large amounts of blood and blood clots. Additionally, it is an invasive method, not exempt of complications such as the risk of intestinal perforation ${ }^{(11,14)}$. According to a comprehensive study undertaken in an emergency context, colonoscopy can identify the definite bleeding source in only $13 \%$ of the patients and the probable source in $67 \%$ of the patients ${ }^{(15)}$.

Capsule endoscopy, on its turn, is utilized for the evaluation of obscure bleeding, but is not feasible in an emergency scenario. Studies have demonstrated that capsule endoscopy can be diagnostic of obscure bleeding in $50-70 \%$ of the patients $^{(16)}$. Its advantages include the capacity of screening the entire small bowel, being more sensitive in those patients presenting with clinically visible bleeding than in those presenting with occult bleeding. However, it does not allow for biopsies to be performed and, like with other endoscopic methods, a massive hemorrhage may impair the visualization of the site of active bleeding ${ }^{(3,16)}$.

Technetium-99m-labeled red cell scintigraphy is a noninvasive method that does not require bowel preparation and detects both arterial and venous bleeding, with a sensitivity of up to $93 \%$ and specificity up to $95 \%$, in the presence of a bleeding rate $\geq 0.4 \mathrm{~mL} / \mathrm{min}^{(10)}$. It allows for image acquisition for an extended period of time, being particularly useful in cases of intermittent bleeding. Its main limitation is the inaccurate definition of the anatomic site of the bleeding. In addition, it is a method that is not always available, requiring a long acquisition time, and difficult to be performed at an emergency unit, particularly during night shifts $^{(3,10)}$.

Digital angiography is a widely utilized method in cases of GIB whenever upper gastrointestinal endoscopy and optical colonoscopy are negative in the identification of the bleeding site or in cases where a therapeutic intervention is required. However, it is an invasive and highly expensive method, requiring specialized professionals, a fact that may limit its availability at certain services and periods. Also, it poses risks of complications inherent to catheterization and may present false-negative results because of anatomical vascular variations. The method detects bleeding at rates $\geq 0.5 \mathrm{~mL} / \mathrm{min}$, with a sensitivity of 63-90\% for upper GIB and $40-86 \%$ for lower GIB, with a specificity that may reach $100 \%{ }^{(3,10,17)}$.

Since its clinical inception, MDCT has demonstrated high spatial and temporal resolutions ${ }^{(18)}$. By means of MDCT, it is possible to perform angiographic studies with multiplanar reconstructions. With that, CT angiography has become a fast, minimally invasive, and widely available method that allows for accurate localization of upper and lower GIB, particularly in the ileum and jejunum, both sites that are hardly accessible at upper digestive endoscopy and optical colonoscopy $^{(3,5)}$. Systematic reviews evaluating the accuracy of CT angiography at GIB have demonstrated sensitivity of $85.2-89.0 \%$ and specificity of $85.0-95.0 \%(17,19,20)$. A study with animal model has demonstrated that CT angiography is capable of detecting bleedings of $0.3 \mathrm{~mL} / \mathrm{min}^{(21)}$.

The advantage of CT angiography over the endovascular procedures is related to its capability of accurately evaluating extraluminal abnormalities, supplying and draining vessels, regional anatomy and disease relationship with adjacent structures. Thus, the appropriate arterial mapping by means of CT angiography, before a therapeutic procedure such as digital angiography, can reduce the intervention time, exposure to radiation and the contrast agent dose, benefiting the patient ${ }^{(3)}$.

The location of the bleeding and the diagnosis of its cause may play an important role in the management and treatment of such patients. For example, the accurate identification of the bleeding site can determine how the endoscopic approach will be performed, especially in those cases where it is difficult to differentiate upper from lower bleeding, with basis on the patient's clinical condition (Figure 1).

CT angiography can accurately determine the four main causes of lower GIB (angiodysplasia, colonic diverticulum, neoplasms and colitis), providing guidance for specific treatment. Thus, the location of the bleeding in the small bowel 


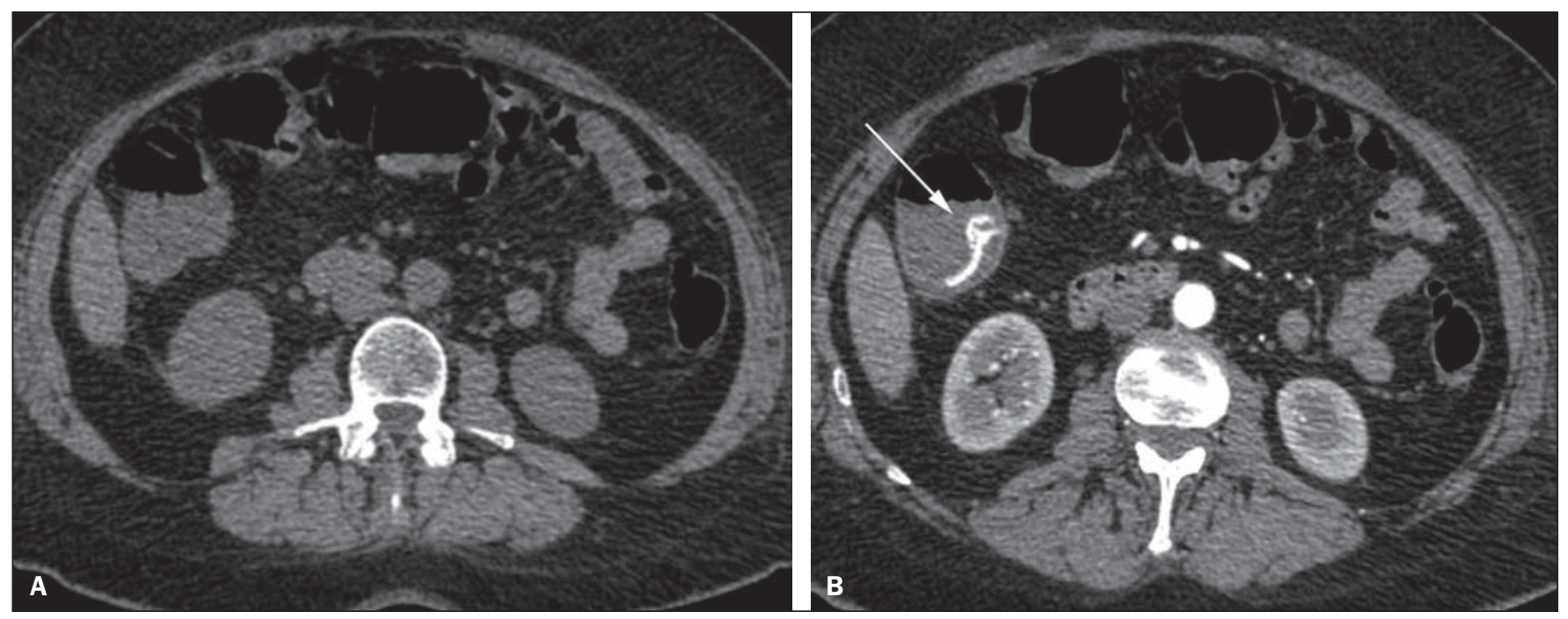

Figure 1. Male, 60-year-old patient. Active bleeding observed in the ascending colon, characterized by increased density with linear appearance in the arterial phase (arrow on B) when compared with the non-contrast-enhanced image (A).

can avoid unnecessary endoscopic examination, bringing forward endovascular or surgical treatment ${ }^{(10)}$.

The differentiation between GIB caused by diverticulum from another caused by angiodysplasia is important, as in untreated angiodysplasia the bleeding may recur in up to $85 \%$ of the patients, contrary to diverticular disease, where $25 \%$ of the patients are affected by recurrence ${ }^{(22,23)}$. The tumors that cause digestive hemorrhage preferably require surgical treatment. When colectomy is performed without previous knowledge of the cause and location of the bleeding, the mortality rates reach $33 \%$ for total colectomy and $57 \%$ for partial colectomy ${ }^{(24)}$.

The literature describes several CT angiography protocols to evaluate GIB, with various technical variables that might influence the effectiveness of the scan ${ }^{(5,13,14,18,25-40)}$ (Table 1). It is not clear yet how such parameters might be combined to obtain images with high accuracy, short acquisition time and limited radiation dose, while avoiding unnecessary acquisition phases. With that in mind, the authors undertook a critical literature review in order to determine, by means of the analysis of adopted technical variables, a scan protocol that can provide the best results in the diagnosis of GIB in patients admitted to the emergency service, offering practical suggestions in order to avoid common error in the scan performance and images interpretation.

\section{VARIABLES INVOLVED IN THE PREPARATION AND PERFORMANCE OF CT ANGIOGRAPHY}

\section{Preparation (fasting and oral contrast)}

Fasting is not indispensable and is frequently unfeasible, as the necessity of the diagnosis originates from an emergency, and the patient might have eaten before admission. In addition, because of the intermittent nature of GIB, it is important that CT angiography be performed as soon as possible, when the active GIB is clinically detected, in order to maximize the diagnostic capability of the $\operatorname{method}^{(10)}$.

At CT angiography, the active bleeding is characterized by intravenous contrast medium extravasation into the bowel lumen $^{(6)}$ (Figure 2). For that reason, the administration of neutral oral contrast medium (for example, water) or positive oral contrast material (for example, 5\% iodine solution) should be avoided, as in the case of water, it can dilute the

Table 1-CT angiography parameters for patients presenting with gastrointestinal bleeding, according to data obtained in the literature and demonstrating wide variability ${ }^{(\mathbf{5 , 1 0}, 11,15,22-37)}$.

\begin{tabular}{lc}
\hline Parameter & Literature \\
\hline Phases & $\begin{array}{c}\text { Arterial / non-contrast enhanced + arterial / non-contrast enhanced + portal / arterial + portal / non-contrast } \\
\text { enhanced + arterial + portal / non-contrast enhanced + arterial + portal + late / arterial + enteric + portal } \\
\text { No / yes (water) } \\
\text { Concentration of intravenous contrast }\end{array}$ \\
Intravenous contrast injection rate & $270-400 \mathrm{mg} / \mathrm{mL}$ \\
Intravenous contrast volume & $3-5 \mathrm{~mL} / \mathrm{s}$ \\
kV & $60-160 \mathrm{~mL}$ \\
mA & $100-140$ \\
Thickness & $180-586$ \\
Number of detectors & $0.6-5.5 \mathrm{~mm}$ \\
Threshold (abdominal aorta) & $4,8,16,40,64$ \\
\hline
\end{tabular}



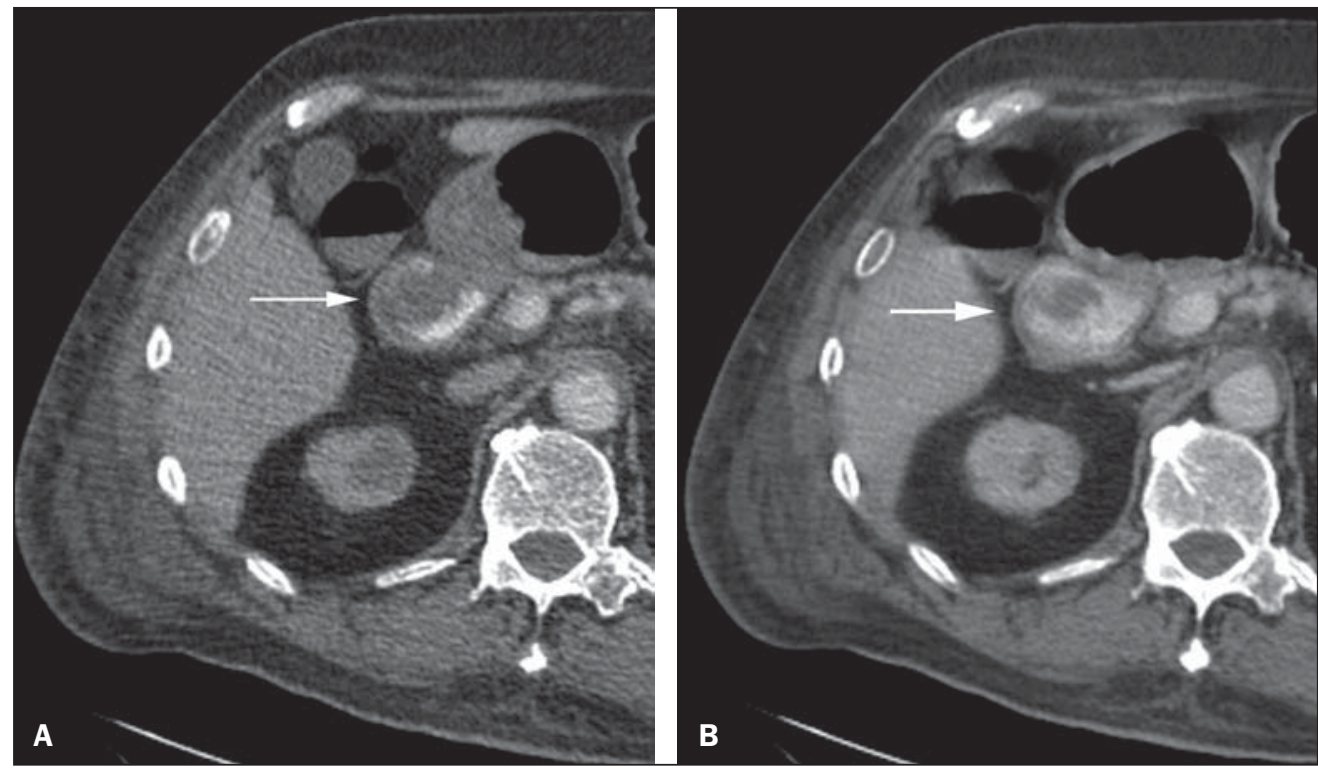

Figure 2. Male, 65-year-old patient presenting with hematemesis. CT angiography in the arterial phase (A) demonstrating active contrast medium extravasation (arrow) in the first portion of the duodenum, which enhances in the portal phase (B)

intravenously injected contrast agent upon its extravasation to the lumen of the bowel loop, thus impairing its detection; on the other hand, the positive contrast medium occupying the bowel lumen will impair the identification of the intravenously injected contrast agent extravasation, leading to a falsenegative result (Figure 3). It is also possible to speed up the performance of the scan avoiding bowel preparation, thus anticipating the diagnosis and the eventually required treatment, with incontrovertible advantages for the patient ${ }^{(6,41)}$.

\section{Technical parameters $(\mathrm{kV}, \mathrm{mAs}$, slice thickness $)$}

The reduction of radiation dose has been a constant preoccupation in the design of CT protocols, by limiting the levels of $\mathrm{kV}$ and $\mathrm{mAs}$, besides reducing the number of CT images acquisition phases as a function of its clinical indication $^{(42,43)}$.

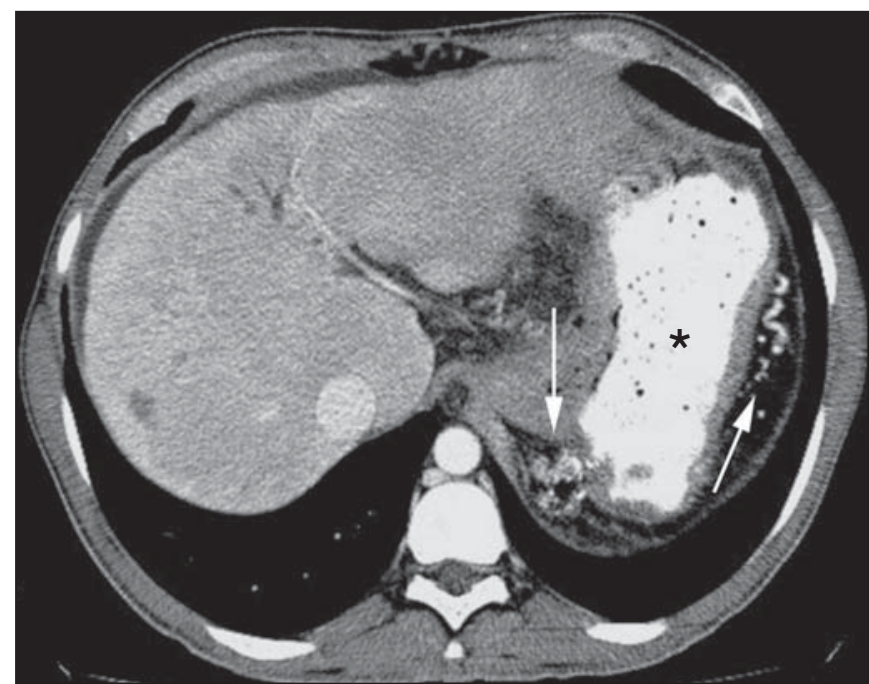

Figure 3. Cirrhotic patient with esophageal varices and hematemesis. In spite of extensive collateral circulation, well identified at CT (arrows), it is not possible to observe a possible active bleeding due to the presence of iodinated contrast medium in the gastric cavity (asterisk).
In that sense, it has already been demonstrated that in the evaluation of the abdominal aorta by means of CT angiography in patients weighting less than $70 \mathrm{~kg}$, the reduction of tube voltage to $90 \mathrm{kV}$ maintains the diagnostic effectiveness of the method, with reduction of the radiation dose $\mathrm{e}^{(4)}$. In another study, the authors have concluded that, as the tube voltage is reduced, the intravenous contrast volume may also be reduced without causing degradation in image quality ${ }^{(45)}$. However, there is no evidence in the literature that the same happens in GIB evaluation. Most articles in the literature approaching the effectiveness of CT angiography in GIB utilized tube voltage $\geq 120 \mathrm{kV}^{(5,14,18,26,29,30,32,33,37-40)}$. Similarly, there are no studies demonstrating the direct impact of milliamperes magnitude and slice thickness on the effectiveness of CT angiography in the diagnosis of GIB. On the other hand, as the current literature is reviewed, the current intensity utilized in the investigation of GIB has significantly varied (Table 1), and it was not possible to establish a recommended minimum mA level. The adoption of radiation dose reduction strategies, among them the utilization of new reconstruction algorithms, such as the iterative ones (for example: ASIR $^{\circledR}$ or iDose ${ }^{\circledR}$ ), should establish new milliampere standards utilized in CT scans, among those, abdominal CT angiography ${ }^{(46-48)}$.

Intravenous contrast medium (concentration, dose, injection rate)

Iodine concentration, contrast agent dose and its injection rate represent variables that undoubtedly influence in some way the diagnostic quality of many CT scans ${ }^{(49,50)}$. However, a consensus is still to be reached in the literature about the influence of the iodine concentration in the intravenous contrast agent on the CT angiography images quality. On the other hand, better designed studies targeted on this question indicate that high density iodinated contrast media (350-400 mgI/mL) provide greater enhancement of 
abdominal arteries than those with lower densities (300-320 $\mathrm{mgI} / \mathrm{mL})^{(51-53)}$.

In abdominal CT, the iodine dose should be $35-45 \mathrm{~g}$ or approximately $1.5-2.0 \mathrm{~mL} / \mathrm{kg}$ of weight (depending upon utilized iodine concentration) and, for abdominal vascular evaluation, such a dose may be reduced to $1.0-1.5 \mathrm{~mL} / \mathrm{kg}^{(54)}$. Considering that, besides a merely vascular evaluation, many GIB patients require visceral evaluation, the contrast medium dose adopted by most reviewed authors is around 1.5-2.0 $\mathrm{mL} / \mathrm{kg}$ (or between 100 and $150 \mathrm{~mL}$ ) (Table 1).

It is known that a high injection rate $(4-5 \mathrm{~mL} / \mathrm{s})$ is important for the acquisition of the arterial phase ${ }^{(55)}$, and most studies in the literature approaching GIB evaluation utilized injection rate $\geq 4 \mathrm{~mL} / \mathrm{s}^{(5,14,25,26,30-35,38,39)}$.

\section{CT phases and acquisition times}

The use of CT angiography in cases of suspicion of GIB has been implemented with a wide variability of protocols (Table 1), but a protocol comprising three acquisition phases (non-contrast-enhanced, arterial and portal phases) has most frequently been adopted ${ }^{(14,25,26,28,29,31,34,35,37,39,40)}$.

There is a consensus about the relevance of utilizing the non-contrast-enhanced phase in order to avoid pitfalls such as suture material, surgical clips, foreign bodies and retained contrast medium $^{(3,6,10)}$. However, one should be careful with the utilized radiation dose, particularly in the case of young patients. For this reason, the utilization of a protocol with low radiation dose for the non-contrast-enhanced phase is recommended ${ }^{(14)}$.

An experimental study demonstrated that the combined utilization of the arterial and portal phases offers higher sensitivity for the detection of small bowel bleeding as compared with the utilization of a single phase ${ }^{(56)}$. The portal phase can improve the accuracy of the arterial phase in detecting and localizing the bleeding, particularly in cases where the bleeding originates from bowel tumors ${ }^{(11)}$. Another study demonstrated that the arterial phase was capable of identifying the site of the bleeding in all positive cases, and the portal phase confirmed the findings, with increased parietal enhancement and/or intraluminal accumulation of iodinated contrast medium, providing greater diagnostic reliability. On the other hand, the adoption of the delayed phase (or equilibrium phase, performed between 3 and 5 minutes after starting the injection of intravenous contrast medium) did not contribute with additional findings and improvement in the CT angiography accuracy in the evaluation of GIB patients $^{(33)}$.

\section{CT ANGIOGRAPHY ANALYSIS AND IMAGING FINDINGS}

The tomographic criterion for the diagnosis of active GIB is the extravasation of the intravenously injected contrast medium to the lumen of the gastrointestinal tract (Figures 1, 2 and 4), and some authors utilize an objective density measurement $>90$ Hounsfield units (HU) within the intestinal lumen ${ }^{(5,29,34,35,38,40)}$. In the authors' experience, as well as other authors' experience ${ }^{(3,10)}$, the simple visual comparison of the pre- and post-intravenous contrast images is enough to confirm the diagnosis, avoiding the already mentioned pitfalls. The contrast medium extravasation may have several appearances, namely: linear, stream, whirl, ellipsoid, or it may also occupy the entire luminal thickness of the bowel loop, and in such case one should be careful not to confuse the intraluminal extravasation of the contrast with the normal mucosal enhancement of the bowel loop, particularly in cases where it is collapsed ${ }^{(3,10)}$. Secondary findings should also be evaluated, and whenever present, they increase the method sensitivity. For example, acute hematoma in the non-contrast-enhanced phase indicates recent bleeding $^{(14)}$ (Figure 5). Other findings that may indicate the
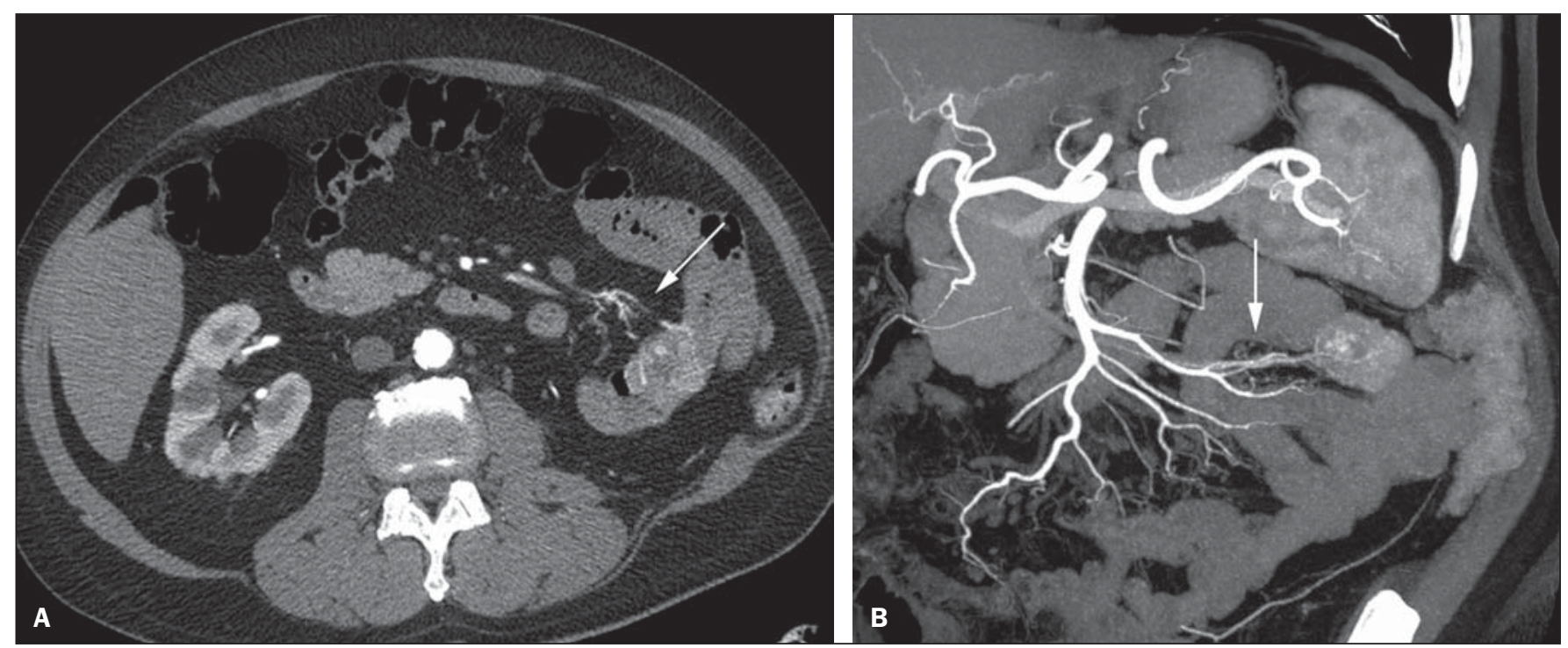

Figure 4. CT angiography in the arterial phase (A) and the corresponding angiographic MIP reconstruction (B). Active bleeding is observed in the jejunum (arrow on A). MIP reconstruction allows for identifying the branch of the superior mesenteric artery as the source of the bleeding (arrow on B). 


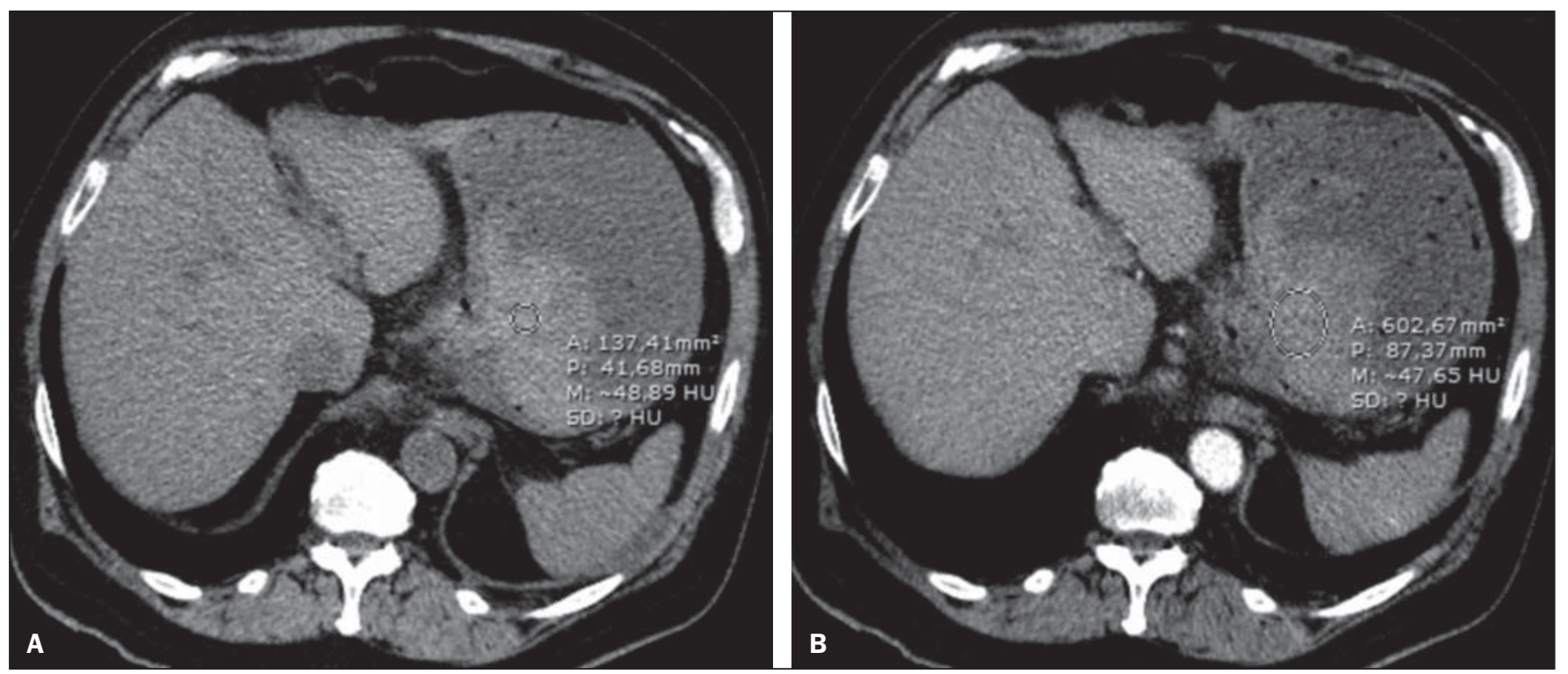

Figure 5. Abdominal CT angiography. Non-contrast-enhanced phase (A) and arterial phase (B). A great amount of blood is observed in the gastric chamber, characterized by hyperdense material in the non-contrast-enhanced phase ( $48 \mathrm{HU})$ and without enhancement in the arterial phase (47 HU). No evidence of active bleeding is observed.

cause of the bleeding and guide the management of such patients include: a) hypoenhancement and/or intestinal parietal thickening; b) presence of tumor lesion (Figure 6); c) vascular anomaly; d) esophageal varices, gastric varices or rectal varices (Figure 7); e) ulcer (Figure 8); f) abnormally enhanced polyp or diverticulum ${ }^{(5,25,31,33-35,38)}$.

The analysis of multiplanar reformation images can enhance the diagnostic capability in acute GIB, particularly in cases of small lesions, such as angiodysplasia and arteriovenous malformations ${ }^{(33)}$. Reformatted coronal maximum intensity projection (MIP) images are useful in the abdominal localization of the intestinal segment with bleeding and in the evaluation of the proximal femur vascularization in cases where angiography is indicated (Figures 4 and 9). On their turn, sagittal reformatted MIP images are useful in the

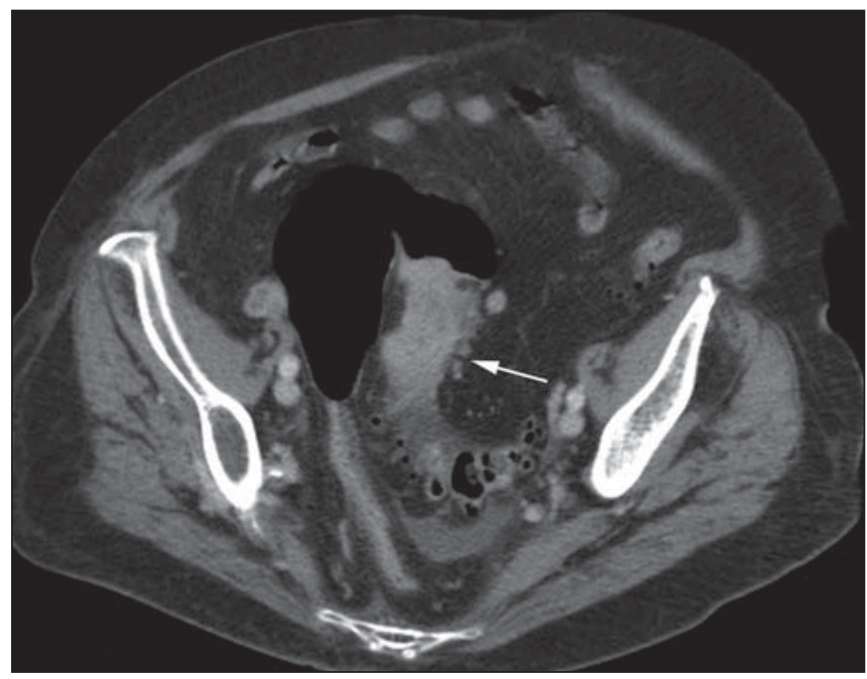

Figure 6. Female, 78-year-old patient presenting with enterorrhagia over the past two days. At CT angiography portal phase, a stenosing lesion is observed in the sigmoid (arrow). Additional finding: diverticular disease in the colon. assessment of the rectum, as well as in the evaluation of the aorta and of the origin of the superior and lower mesenteric $\operatorname{arteries}^{(10)}$.

Hemorrhagic colonic diverticulosis is a frequent cause of lower GIB, commonly involving the ascending colon, while the descending colon and the sigmoid involvement is more frequently associated with inflammatory/infectious complications. Its diagnosis dispense with an accurate localization of the bleeding diverticulum, and the presence of isolated diverticulum with no evidence of active bleeding is not enough to attribute the cause of the bleeding to the diverticular disease ${ }^{(57)}$ (Figure 6).

In the authors' service, as tomographic images from a patient presenting with GIB, one seeks to identify the following signs: a) contrast extravasation to the gastrointestinal tract

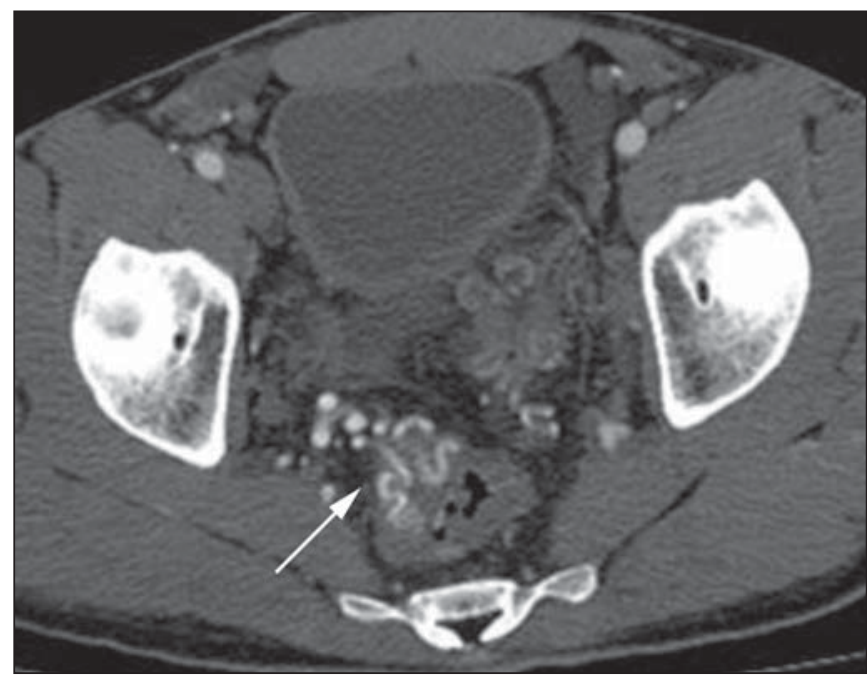

Figure 7. CT angiography, arterial phase in cirrhotic patient, with portal thrombosis and rectal bleeding caused by rectal and sigmoid varices (arrow). 


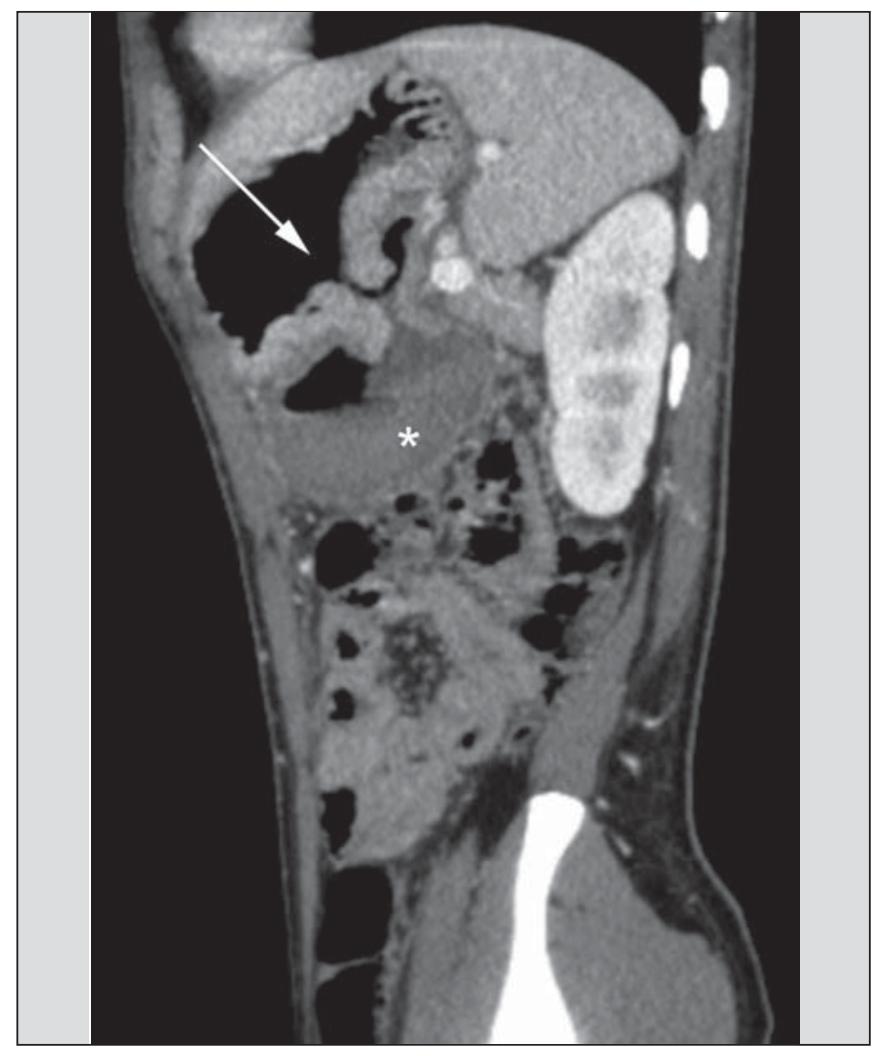

Figure 8. A 19-year-old hemophilia patient with abdominal pain over the past three days and hematemesis. Contrast-enhanced CT in the portal phase demonstrates the presence of a large ulcer in the greater gastric curvature (arrow) communicating the stomach lumen with retrogastric liquid collection with increased density, suggesting hematic origin (asterisk). There is no evidence of active bleeding. Upper digestive endoscopy, performed after CT, demonstrated the presence of extensive ulcerated lesion on the posterior wall of the gastric body, measuring approximately $7.0 \mathrm{~cm}$, with fibrin in the base, and a large orifice $(4.0 \mathrm{~cm}$ diameter) communicating with a large cavity, constituted of walls covered by blood and clots. Histological study of the lesion did not reveal any signs of neoplasia.

lumen; b) increase of such an extravasation in the portal phase; c) abnormal parietal enhancement (hypoenhancement), indicating ischemia or loop distress; d) intestinal wall thickening (>3 mm); e) acute hematoma characterized by hyperattenuating area in the non-contrast-enhanced phase, either with or without enhancement after intravenous injection of the contrast medium; f) presence of tumor lesion in the gastrointestinal wall; g) signs of other GIB causes, such as vascular malformation, abnormally enhanced polyp or diverticulum and presence of ulcer.

\section{PROPOSAL OF CT PROTOCOL}

A specific protocol for patients with a history of active GIB (Table 2) is proposed with basis on the literature review and on the authors' own experience. Initially, one should remember that, because of the clinical risk, hemodynamically unstable patients should not be submitted to CT angiography, prompting the performance of angiography or upper digestive endoscopy because of its therapeutic capability. In cases of stable patients, and considering that even in such cases it is necessary to perform CT angiography as soon as possible, the present study authors do not request patients to fast prior to the scan. Oral contrasts of any kind are not utilized either, in order not to dilute or mask possible intraluminal extravasation of the contrast medium, and to avoid delays in performing the scan. Abdominal CT angiography images are acquired in a 64 -chanel Brilliance $64^{\circledR}$ apparatus (Philips Medical Systems; Cleveland, OH), according to the following parameters: a) detector configuration: $64 \times 0.625$ $\mathrm{mm}$; b) slice thickness: $1 \mathrm{~mm}$; c) reconstruction thickness:

Table 2-Proposed CT angiography protocol for abdomen and pelvis for patients presenting with active gastrointestinal bleeding. Equipment: 64-channel multidetector CT apparatus.

\begin{tabular}{lc}
\hline Parameter & Proposed \\
\hline Phases & $\begin{array}{c}\text { Non-contrast enhanced }+ \text { arterial } \\
+ \text { portal }\end{array}$ \\
Oral contrast & No \\
Intravenous contrast concentration & $350 \mathrm{mg} / \mathrm{mL}$ \\
Intravenous contrast injection rate & $4 \mathrm{~mL} / \mathrm{s}$ \\
Intravenous contrast volume & $100 \mathrm{~mL}$ or $1.5 \mathrm{~mL} / \mathrm{kg}$ \\
kV & 120 \\
mA & Automatic \\
Thickness & $1 \mathrm{~mm}$ \\
Threshold (abdominal aorta) & $100 \mathrm{HU}$ \\
Time for arterial phase initiation & 20 seconds after threshold \\
Time for portal phase initiation & 40 seconds after threshold \\
\hline
\end{tabular}
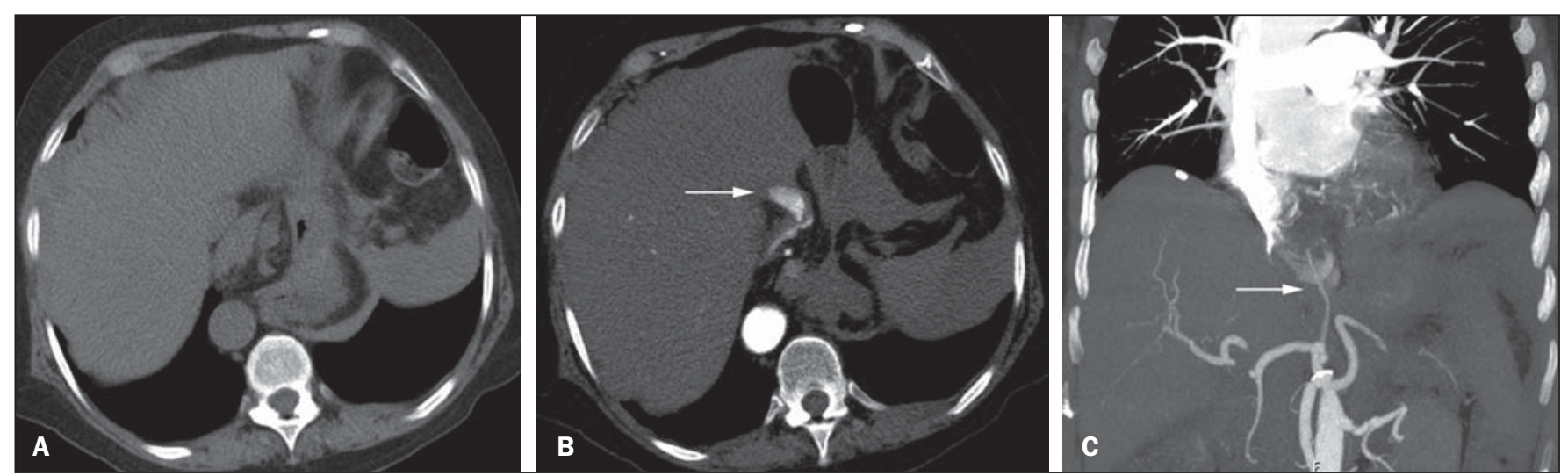

Figure 9. Non-contrast-enhanced CT angiography (A), in the arterial phase (B) and MIP reconstruction (C). Signs of active bleeding, with contrast medium extravasation (arrow on A) coming from the left gastric artery (arrow on $\mathbf{C}$ ). 
1 and $3 \mathrm{~mm}$; d) $120 \mathrm{kV}$; e) mAs depending upon the automatic modulation of the radiation dose (DoseRight ACS $^{\circledR}$ ); f) pitch of $0.67 ; \mathrm{g}$ ) rotation time of 0.5 second. The acquisition time of the arterial phase is approximately 12 seconds, depending upon patient's dimensions.

Considering the relevance of the rational utilization of radiation, the non-contrast-enhanced phase is acquired with a low-dose protocol. A volume of $120 \mathrm{~mL}$ of non-ionic iodinated contrast medium is intravenously injected, preferably into the antecubital vein, by means of an automated Injektron $82 \mathrm{CT} / \mathrm{DG}^{\circledR}$ (Guerbet) injection pump at a rate of $4 \mathrm{~mL} / \mathrm{s}$, followed by $30 \mathrm{~mL}$ saline solution flush, at the same injection rate. Arterial and portal phases acquisition is performed as they are complementary and, as previously mentioned, increase the overall effectiveness of the scan. The beginning of the arterial phase acquisition is defined by means of the automated bolus triggering technique that defines a circular region of interest within the abdominal aorta, immediately above the celiac trunk. The arterial phase initiates $20 \mathrm{sec}-$ onds after a density of $100 \mathrm{HU}$ is achieved in the descending aorta. The portal phase initiates 40 seconds after such a peak. Once a fixed time is established, one may adopt 40and 70-second spans after starting the intravenous contrast injection, in order to obtain the arterial and portal phases respectively. One should consider that delaying the arterial acquisition time is less detrimental than anticipating it, as a longer interval allows for the contrast medium to cross the thin vascular network of the intestinal wall and reach its lumen, in cases of active bleeding. However, it is important to observe that better results are obtained when the automated bolus tracking program is utilized.

The images analysis initiates by the non-contrast-enhanced phase, by seeking to identify spontaneously hyperattenuating spots in the intestinal lumen that might represent pitfalls in the contrast-enhanced phases and the presence of high density areas on the intestinal wall suggesting the diagnosis of acute hematoma (Figure 10). Subsequently, such images are carefully compared with those obtained in the arterial and portal phases, seeking to identify areas of contrast medium extravasation in the gastrointestinal lumen (Figure 10) and secondary signs as the above mentioned ones. Finally, by means of multiplanar angiographic reconstructions with the MIP technique, one seeks to identify with greater accuracy not only the origin of the bleeding, but also, whenever possible, the supplying vessel (Figures 4 and 9) and possible vascular anatomic variants. Such data may be useful in the planning and performance of therapeutic angiography.

\section{CONCLUSION}

GIB is a frequent medical emergency, with important morbimortality rates, requiring a fast diagnostic tool capable of localizing the site and cause of the bleeding, and consequently allowing for the institution of appropriate treatment as soon as possible. Abdominal CT angiography is a fast, minimally invasive and widely available method that can precisely and accurately determine the location and cause of digestive hemorrhage. The effectiveness of the method is optimized with the implementation of a scan protocol that takes into consideration the several technical variables that somehow influence the detection of the bleeding site without neglecting factors that might limit the radiation dose. Based on such considerations, the present study authors suggest that for hemodynamically stable patients presenting with active GIB, CT angiography be performed as first-line diagnostic tool as soon as possible, in order to maximize the bleeding detection capability and assist in the therapeutic planning.

\section{REFERENCES}

1. Manning-Dimmitt LL, Dimmitt SG, Wilson GR. Diagnosis of gastrointestinal bleeding in adults. Am Fam Physician. 2005;71:1339_ 46.

2. Bezerra KB, Bacelar Jr EA, Pereira NCS, et al. Gastric arteriovenous malformation: treatment by embolization. Radiol Bras. 2012;45: 126-8.

3. Geffroy Y, Rodallec MH, Boulay-Coletta I, et al. Multidetector CT angiography in acute gastrointestinal bleeding: why, when, and how Radiographics. 2011;31:E35-46.

4. Singh V, Alexander JA. The evaluation and management of obscure
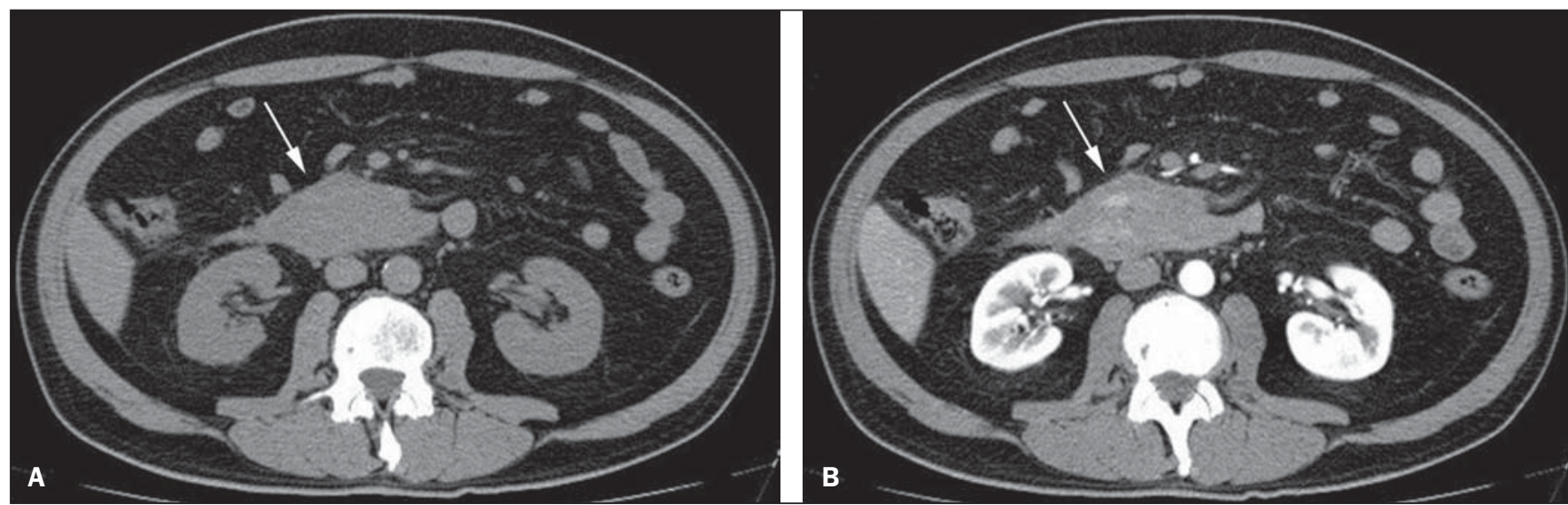

Figure 10. Posterior wall ulcer in the third portion of the duodenum with signs of active bleeding characterized by contrast medium extravasation identified by difference in density between non-contrast-enhanced phase (A) and contrast-enhanced phase (B) (arrows) and by the enhancement appearance. 
and occult gastrointestinal bleeding. Abdom Imaging. 2009;34: $311-9$.

5. Jaeckle T, Stuber G, Hoffmann MH, et al. Detection and localization of acute upper and lower gastrointestinal (GI) bleeding with arterial phase multi-detector row helical CT. Eur Radiol. 2008;18: 1406-13.

6. Stuber T, Hoffmann MH, Stuber G, et al. Pitfalls in detection of acute gastrointestinal bleeding with multi-detector row helical CT. Abdom Imaging. 2009;34:476-82.

7. Barbano PR, Coviello D, Mariani M, et al. Hemorrhages of the upper gastrointestinal tract. A comparison of the diagnostic accuracy between endoscopic and radiological examinations. Minerva Chir. 1990;45:1003-8.

8. Silverstein FE, Gilbert DA, Tedesco FJ, et al. The national ASGE survey on upper gastrointestinal bleeding. I. Study design and baseline data. Gastrointest Endosc. 1981;27:73-9.

9. Salvador Añez M, González Guerra H, Pacheco JL, et al. Value of emergency endoscopy in upper digestive hemorrhage. G E N. 1990; $44: 125-33$

10. Laing CJ, Tobias T, Rosenblum DI, et al. Acute gastrointestinal bleeding: emerging role of multidetector CT angiography and review of current imaging techniques. Radiographics. 2007;27:105570 .

11. Jaeckle T, Stuber G, Hoffmann MH, et al. Acute gastrointestinal bleeding: value of MDCT. Abdom Imaging. 2008;33:285-93.

12. Vreeburg EM, Snel P, de Bruijne JW, et al. Acute upper gastrointestinal bleeding in the Amsterdam area: incidence, diagnosis, and clinical outcome. Am J Gastroenterol. 1997;92:236-43.

13. Frattaroli FM, Casciani E, Spoletini D, et al. Prospective study comparing multi-detector row CT and endoscopy in acute gastrointestinal bleeding. World J Surg. 2009;33:2209-17.

14. Martí M, Artigas JM, Garzón G, et al. Acute lower intestinal bleeding: feasibility and diagnostic performance of CT angiography. Radiology. 2012;262:109-16.

15. Angtuaco TL, Reddy SK, Drapkin S, et al. The utility of urgent colonoscopy in the evaluation of acute lower gastrointestinal tract bleeding: a 2-year experience from a single center. Am J Gastroenterol. 2001;96:1782-5.

16. Graça BM, Freire PA, Brito JB, et al. Gastroenterologic and radiologic approach to obscure gastrointestinal bleeding: how, why, and when? Radiographics. 2010;30:235-52.

17. García-Blázquez V, Vicente-Bártulos A, Olavarria-Delgado A, et al. Accuracy of CT angiography in the diagnosis of acute gastrointestinal bleeding: systematic review and meta-analysis. Eur Radiol. 2013;23:1181-90.

18. Yoon W, Jeong YY, Shin SS, et al. Acute massive gastrointestinal bleeding: detection and localization with arterial phase multi-detector row helical CT. Radiology. 2006;239:160-7.

19. Chua AE, Ridley LJ. Diagnostic accuracy of CT angiography in acute gastrointestinal bleeding. J Med Imaging Radiat Oncol. 2008, $52: 333-8$

20. Wu LM, Xu JR, Yin Y, et al. Usefulness of CT angiography in diagnosing acute gastrointestinal bleeding: a meta-analysis. World J Gastroenterol. 2010;16:3957-63.

21. Kuhle WG, Sheiman RG. Detection of active colonic hemorrhage with use of helical CT: findings in a swine model. Radiology. 2003 228:743-52.

22. Elta GH. Approach to the patient with gross gastrointestinal bleeding. In: Yamada T, editor. Textbook of gastroenterology. 4th ed. Philadelphia, PA: Lippincott Williams \& Wilkins; 2003. p. 698723.

23. Schuetz A, Jauch KW. Lower gastrointestinal bleeding: therapeutic strategies, surgical techniques and results. Langenbecks Arch Surg. 2001;386:17-25.

24. Zuckerman GR, Prakash C, Askin MP, et al. AGA technical review on the evaluation and management of occult and obscure gastrointestinal bleeding. Gastroenterology. 2000;118:201-21.

25. Ernst O, Bulois $\mathrm{P}$, Saint-Drenant $\mathrm{S}$, et al. Helical CT in acute lower gastrointestinal bleeding. Eur Radiol. 2003;13:114-7.

26. Miller FH, Hwang CM. An initial experience: using helical CT imaging to detect obscure gastrointestinal bleeding. Clin Imaging. 2004;28:245-5 1

27. Tew K, Davies RP, Jadun CK, et al. MDCT of acute lower gastrointestinal bleeding. AJR Am J Roentgenol. 2004;182:427-30.

28. Sabharwal R, Vladica P, Chou R, et al. Helical CT in the diagnosis of acute lower gastrointestinal haemorrhage. Eur J Radiol. 2006; 58:273-9.

29. Scheffel H, Pfammatter T, Wildi S, et al. Acute gastrointestinal bleeding: detection of source and etiology with multi-detector-row CT. Eur Radiol. 2007;17:1555-65.

30. Zink SI, Ohki SK, Stein B, et al. Noninvasive evaluation of active lower gastrointestinal bleeding: comparison between contrast-enhanced MDCT and ${ }^{99 \mathrm{~m}} \mathrm{Tc}$-labeled RBC scintigraphy. AJR Am J Roentgenol. 2008;191:1107-14.

31. Lee S, Welman CJ, Ramsay D. Investigation of acute lower gastrointestinal bleeding with 16- and 64-slice multidetector CT. J Med Imaging Radiat Oncol. 2009;53:56-63.

32. Kennedy DW, Laing CJ, Tseng LH, et al. Detection of active gastrointestinal hemorrhage with CT angiography: a 4(1/2)-year retrospective review. J Vasc Interv Radiol. 2010;21:848-55.

33. Al-Saeed O, Kombar O, Morsy M, et al. Sixty-four multi-detector computerised tomography in the detection of lower gastrointestinal bleeding: a prospective study. J Med Imaging Radiat Oncol. 2011;55:252-8.

34. Chang WC, Tsai SH, Chang WK, et al. The value of multidetectorrow computed tomography for localization of obscure acute gastrointestinal bleeding. Eur J Radiol. 201 1;80:229-35.

35. Sun H, Jin Z, Li X, et al. Detection and localization of active gastrointestinal bleeding with multidetector row computed tomography angiography: a 5-year prospective study in one medical center. J Clin Gastroenterol. 2012;46:31-41.

36. Yen HH, Chen YY, Yang CW, et al. Clinical impact of multidetector computed tomography before double-balloon enteroscopy for obscure gastrointestinal bleeding. World J Gastroenterol. 2012;18 $692-7$.

37. Fung HS, Kwok PC, Lau S, et al. 64-Slice multi-detector computed tomography for detection of acute gastrointestinal bleeding. J HK Coll Radiol. 2008;1 1:13-8.

38. Heiss P, Zorger N, Hamer OW, et al. Optimized multidetector computed tomographic protocol for the diagnosis of active obscure gastrointestinal bleeding: a feasibility study. J Comput Assist Tomogr. 2009;33:698-704.

39. Palma J, Mihaila M, Pilleul F. Multidetector computed tomography in acute lower gastrointestinal bleeding. Reports in Medical Imaging. 2010;3:107-13.

40. Kim JW, Shin SS, Yoon W, et al. Diagnosis of acute gastrointestinal bleeding: comparison of the arterial, the portal, and the combined set using 64-section computed tomography. J Comput Assist Tomogr. 2011 1;35:206-11.

41. Horton KM, Jeffrey RB, Federle MP, et al. Acute gastrointestinal bleeding: the potential role of 64 MDCT and 3D imaging in the diagnosis. Emerg Radiol. 2009;16:349-56.

42. Costa DMC, Salvadori PS, Monjardim RF, et al. When the noncontrast-enhanced phase is unnecessary in abdominal computed tomography scans? A retrospective analysis of 244 cases. Radiol Bras. 2013;46:197-202

43. Salvadori PS, Costa DMC, Romano RFT, et al. What is the real role of the equilibrium phase in abdominal computed tomography? Radiol Bras. 2013;46:65-70.

44. Nakayama Y, Awai K, Funama Y, et al. Lower tube voltage reduces 
contrast material and radiation doses on 16-MDCT aortography. AJR Am J Roentgenol. 2006;187:W490-7.

45. Nakayama Y, Awai K, Funama Y, et al. Abdominal CT with low tube voltage: preliminary observations about radiation dose, contrast enhancement, image quality, and noise. Radiology. 2005;237: 945-51.

46. Suzuki S, Nishiyama Y, Kuwahara S, et al. Adaptive statistical iterative reconstruction algorithm for measurement of vascular diameter on computed tomographic angiography in vitro. J Comput Assist Tomogr. 2013;37:311-6.

47. Martillotti J, Silva N, Chhabra J, et al. Dose of reduced z-axis length of computed tomography angiography (CTA) of the chest for pulmonary embolism using 64-detector rows and adaptive iterative reconstruction techniques. Emerg Radiol. 2013;20:39-44.

48. Cornfeld D, Israel G, Detroy E, et al. Impact of Adaptive Statistical Iterative Reconstruction (ASIR) on radiation dose and image quality in aortic dissection studies: a qualitative and quantitative analysis. AJR Am J Roentgenol. 2011;196:W336-40.

49. Rubin GD. Techniques for performing multidetector-row computed tomographic angiography. Tech Vasc Interv Radiol. 2001;4:2-14.

50. Capuñay C, Carrascosa P, Martín López E, et al. Multidetector CT angiography and virtual angioscopy of the abdomen. Abdom Imaging. 2009;34:81-93.

51. Loewe C, Becker CR, Berletti R, et al. 64-Slice CT angiography of the abdominal aorta and abdominal arteries: comparison of the di- agnostic efficacy of iobitridol $350 \mathrm{mgI} / \mathrm{ml}$ versus iomeprol $400 \mathrm{mgI} /$ $\mathrm{ml}$ in a prospective, randomised, double-blind multi-centre trial. Eur Radiol. 2010;20:572-83.

52. Behrendt FF, Mahnken AH, Keil S, et al. Contrast enhancement in multidetector-row computed tomography (MDCT) of the abdomen: intraindividual comparison of contrast media containing $300 \mathrm{mg}$ versus $370 \mathrm{mg}$ iodine per ml. Eur Radiol. 2008;18:1199205.

53. Sahani DV, Soulez G, Chen KM, et al. A comparison of the efficacy and safety of iopamidol-370 and iodixanol-320 in patients undergoing multidetector-row computed tomography. Invest Radiol. 2007;42:856-61.

54. Hammerstingl RM, Vogl TJ. Abdominal MDCT: protocols and contrast considerations. Eur Radiol. 2005;15 Suppl 5:E78-90.

55. Brink JA. Contrast optimization and scan timing for single and multidetector-row computed tomography. J Comput Assist Tomogr. 2003;27 Suppl 1:S3-8.

56. Dobritz M, Engels HP, Schneider A, et al. Detection of intestinal bleeding with multi-detector row CT in an experimental setup. How many acquisitions are necessary? Eur Radiol. 2009;19:2862-9.

57. Duchat F, Soyer P, Boudiaf M, et al. Multi-detector row CT of patients with acute intestinal bleeding: a new perspective using multiplanar and MIP reformations from submillimeter isotropic voxels. Abdom Imaging. 2010;35:296-305. 